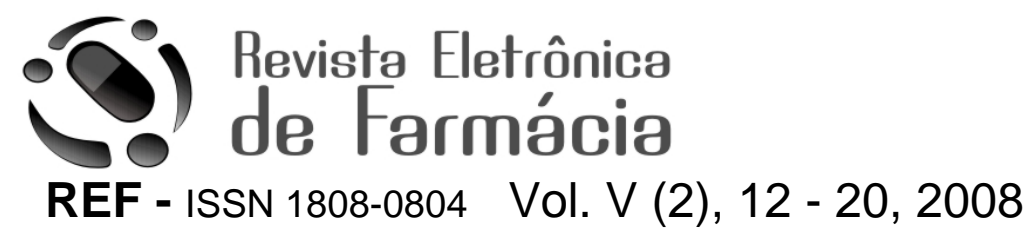

\title{
DIABETES - UTILIZAÇÃO DE PLANTAS MEDICINAIS COMO FORMA OPCIONAL DE TRATAMENTO
}

\author{
DIABETES - UTILIZATION OF MEDICINAL PLANTS \\ AS AN OPTIONAL FORM OF TREATMENT
}

\author{
Borges, K. B. ${ }^{1}$; Bautista, H. B. ${ }^{1}$; Guilera, S. ${ }^{2}$ \\ ${ }^{1}$ Departamento de Ciências da Vida, Universidade do Estado da Bahia, Campus I (UNEB). Rua Silveira Martins, \\ 2555, Cabula. 41.195-001. Salvador-BA, Brasil. \\ ${ }^{2}$ Instituto Brasileiro de Geografia e Estatística (IBGE). Av. Marechal Castelo Branco, 750. 40.046-900. Salvador- \\ BA, Brasil.
}

Autor para correspondência e-mail: kborges@uneb.br

Recebido em 21/05/2008 - Aceito em 06/11/2008

RESUMO: O diabetes é, atualmente, uma das doenças mais importantes que afetam a humanidade. A Organização Mundial de Saúde tem estimulado a investigação de plantas medicinais para serem usadas em seu tratamento, uma vez que essa doença alcançará 300 milhões de pessoas em 2030. Os fitoterápicos podem atuar como forma opcional de terapêutica, considerando-se seu menor custo, cujos benefícios somam-se aos da terapia convencional. Inúmeras plantas tiveram seu efeito hipoglicêmico confirmado experimentalmente, entretanto, muitas não foram validadas como medicinais, via protocolos científicos. O trabalho objetiva identificar plantas medicinais utilizadas no Brasil como antidiabéticas, destacando algumas que tiveram efeitos hipoglicemiantes cientificamente comprovados. Foram relacionados 65 táxons, pertencentes a 35 famílias. Pelo levantamento bibliográfico constatou-se que Arctium minus (Hill) Bernh. (bardana), Eucaliptus globulus Labill. (eucalipto), Syzygium jambolanum DC. (jambolão), Bidens pilosa L. (picão), Salvia officinalis L. (sálvia) foram citadas 7vezes; Urtica spp. (urtigas) 9 vezes; Allium sativum L. (alho), Phyllanthus niruri L. (quebra-pedra) 10 vezes; Baccharis trimera DC. (carqueja) e Anacardium occidentale L. (cajueiro) 11 vezes, e, Bauhinia forficata Link. (pata-de-vaca), 17 vezes. A folha foi o órgão mais utilizado nas preparações. Estudos demonstraram que extratos de 'bardana', 'alho' e 'carqueja' mostraram-se eficientes como terapia complementar em diabéticos. Experimentos apontam para a validade do uso de 'pata-de-vaca' nesse tratamento. Estudo clínico mostrou o efeito hipoglicemiante da casca do 'cajueiro'. Os resultados demonstram a importância dos fitomedicamentos como coadjuvantes no tratamento do diabetes tipo 2. Pesquisas que comprovem a ação terapêutica de princípios ativos vegetais são fundamentais para garantir eficácia e segurança da sua utilização, por profissionais de saúde.

PALAVRAS-CHAVE: Plantas medicinais, diabetes mellitus, Etnobotânica.

ABSTRACT: Diabetes is, nowadays, one of the most important diseases that affect humanity. World Health Organization has stimulated the research of medicinal plants to be used in diabetes treatment once this disease will affect around $\mathbf{3 0 0}$ million people. Fitotherapy can act as optional form of therapy, considering its lower cost, and its benefits can be added to conventional therapy. Innumerable plants had their hipoglicemic effects experimentally proven. However, many of them had not been validated as medicinal by scientific protocols. This paper objective to identify medicinal plants used in Brazil as antidiabetic, detaching some of them that had had hipoglicemic effect scientifically proven. 65 taxa have been related, which belong to 35 families. By the bibliographical survey one evidenced that Arctium minus (Hill) Bernh. (bardana), Eucalyptus globulus Labill. (eucalipto), Syzygium jambolanum DC. (jambolão), Bidens pilosa L. (picão), Salvia officinalis L. (salvia) had been cited 7 times; Urtica spp. (urtiga), 9 times; Allium sativum L. (garlic), Phyllanthus niruri L. (quebra-pedra) 10 times; Baccharis trimera DC. (carqueja) and Anacardium occidentale L. (cashew tree) 11 times; and Bauhinia forficata Link. (pata-de-vaca), 17 times. The leaf was the most used organ in the preparations. Studies had demonstrated that extracts of 'bardana', 'garlic' and 'carqueja' had revealed efficient as complementary therapy in diabetic. Experiments point with respect to the validity of the use of 'pata-de-vaca' in this treatment. Clinical study showed the hipoglicemic effect of the bark of 'cashew tree'. The results demonstrate the importance of phytomedicine as helping in the 
Borges, K. B. et al./Revista Eletrônica de Farmácia Vol 5(2), 12-20, 2008.

treatment of diabetes type 2 . Research that proves the therapeutically action of vegetal active principles is basic to guarantee effectiveness and security on its use by health professionals.

KEYS-WORDS: Medicinal plants, diabetes mellitus, Ethnobotany.

\section{INTRODUÇÃO}

O diabetes, caracterizado por elevados níveis de glicose no sangue, é, atualmente, uma das doenças mais importantes que afetam a humanidade. A Organização Mundial de Saúde (OMS) tem estimulado, nas últimas décadas, a investigação de plantas medicinais para o tratamento desta enfermidade, visto que, de acordo com a Sociedade Brasileira de Diabetes (SBD), em 2002, existiam cerca de 173 milhões de diabéticos no mundo, e a projeção é atingir 300 milhões de diabéticos em 2030 (SOCIEDADE BRASILEIRA DE DIABETES, 2008).

No Brasil, devido ao aumento crescente da população de terceira idade, a demanda por terapias menos onerosas para o tratamento de enfermidades crônico-degenerativas, representa um ganho importante nos investimentos humanos e financeiros empregados na área de saúde. O uso de fitoterápicos pretende atuar como uma forma opcional de terapêutica, disponível aos profissionais que cuidam de pacientes diabéticos, considerando-se ser este um tratamento de menor custo, cujos benefícios se somam aos da terapia convencional.

Inúmeras plantas tiveram seu efeito hipoglicêmico confirmado experimentalmente, entretanto, muitas não foram validadas como medicinais via protocolos científicos, relativos a controle de qualidade e grau de toxidade. Desta forma, a maioria não pode ser aceita como medicamento ético de prescrição livre (VOLPATO et al, 2002; NEGRI, 2005).

Observa-se um descrédito por parte dos profissionais de saúde quanto ao potencial terapêutico dos vegetais tidos popularmente como hipoglicemiantes. Entretanto, muitos dos seus pacientes persistem na sua utilização, muitas vezes associada à medicação tradicional, o que pode resultar em sérios riscos à saúde dos usuários, via potencialização de efeitos ou toxidade.

O trabalho objetiva catalogar as plantas medicinais amplamente utilizadas no Brasil como antidiabéticas, em fontes bibliográficas, em particular, no período de 1986 a 2002, destacando algumas que tiveram seus efeitos hipoglicemiantes cientificamente comprovados.

\section{METODOLOGIA}

Realizou-se um levantamento em 45 fontes bibliográficas, principalmente do período de 1986 a 2002 , relacionadas às áreas de Botânica, Química e Farmacologia. As informações obtidas foram sistematizadas em um banco de dados que geraram uma lista dos nomes vernaculares, epítetos científicos e famílias botânicas com indicação antidiabética. Foram também identificados, os autores (bibliografias) que forneceram as indicações de tais plantas e quantas citações desses nomes ocorreram nos trabalhos referidos (referências bibliográficas).

A nomenclatura dos nomes científicos foi revista e atualizada segundo as bibliografias específicas da Sistemática Vegetal.

\section{RESULTADOS E DISCUSSÃO}

$\mathrm{Na}$ literatura consultada, constatou-se que são utilizadas como antidiabéticas, 65 táxons vegetais, que pertencem a 35 famílias e 63 gêneros. Entre as famílias destacam-se Fabaceae, Myrtaceae e Asteraceae (Figura 01).

Verificou-se que um pequeno grupo formado por cerca de 11 táxons (espécies) é amplamente reconhecido pelas suas propriedades terapêuticas hipoglicemiantes. Pelo levantamento bibliográfico realizado, nesse grupo, destacam-se Arctium minus (Hill) Bernh. (bardana), Eucalyptus globulus Labill. (eucalipto), Syzygium jambolanum DC. (jambolão), Bidens pilosa L. (picão) e Salvia officinalis L. (sálvia) que foram citados 7 vezes; Urtica spp. (urtiga) 9 vezes; Allium sativum L. (alho) e Phyllanthus niruri L. (quebra-pedra) 10 vezes; Baccharis trimera DC. (carqueja) e Anacardium occidentale L. (cajueiro) 11 vezes, e, Bauhinia forficata Link. (pata-de-vaca) 17 vezes (Figura 02).

A folha foi o órgão vegetal mais utilizado nas preparações (38\%), seguida pela raiz e fruto (14\%); toda a planta e a semente (10\%) e caule (6\%). Os demais órgãos obtiveram percentuais inferiores a 6\% (Figura 03). 
Borges, K. B. et al./Revista Eletrônica de Farmácia Vol 5(2), 12-20, 2008.

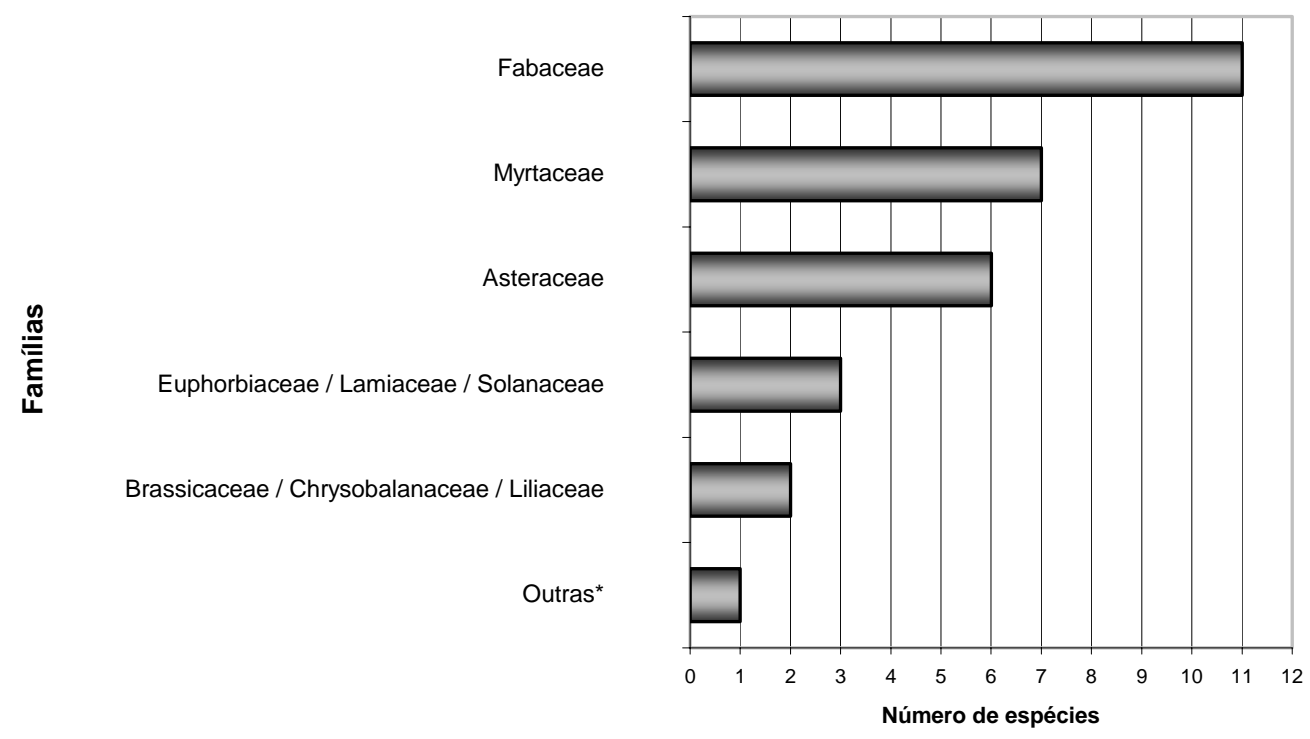

Outras*: Amaranthaceae, Anacardiaceae, Anonaceae, Araceae, Araliaceae, Bignoniaceae, Caprifoliaceae, Cecropiaceae, Clusiaceae, Costaceae, Cucurbitaceae, Dioscoreaceae, Juglandaceae, Oxalidaceae, Plantaginaceae, Poaceae, Portulacaceae, Rubiaceae, Rutaceae, Sapotaceae, Scrophulariaceae, Turneraceae, Urticaceae, Verbenaceae, Vitaceae, Zingiberaceae.

Figura 01 - Famílias e respectivos número de espécies utilizadas como hipoglicemiantes

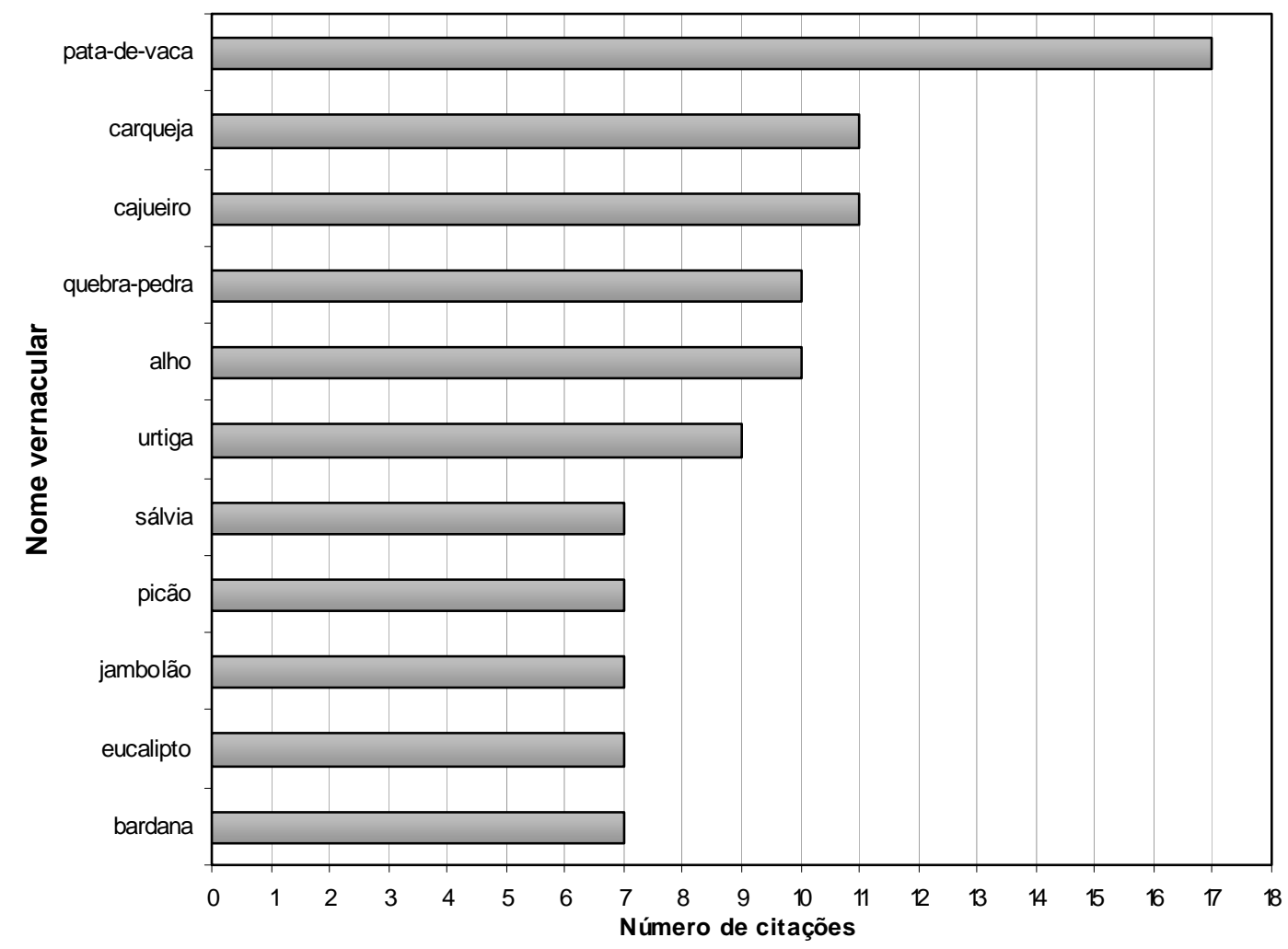

Figura 02 - Número de citações das espécies na bibliografia consultada (ver referências) 
Borges, K. B. et al./Revista Eletrônica de Farmácia Vol 5(2), 12-20, 2008.

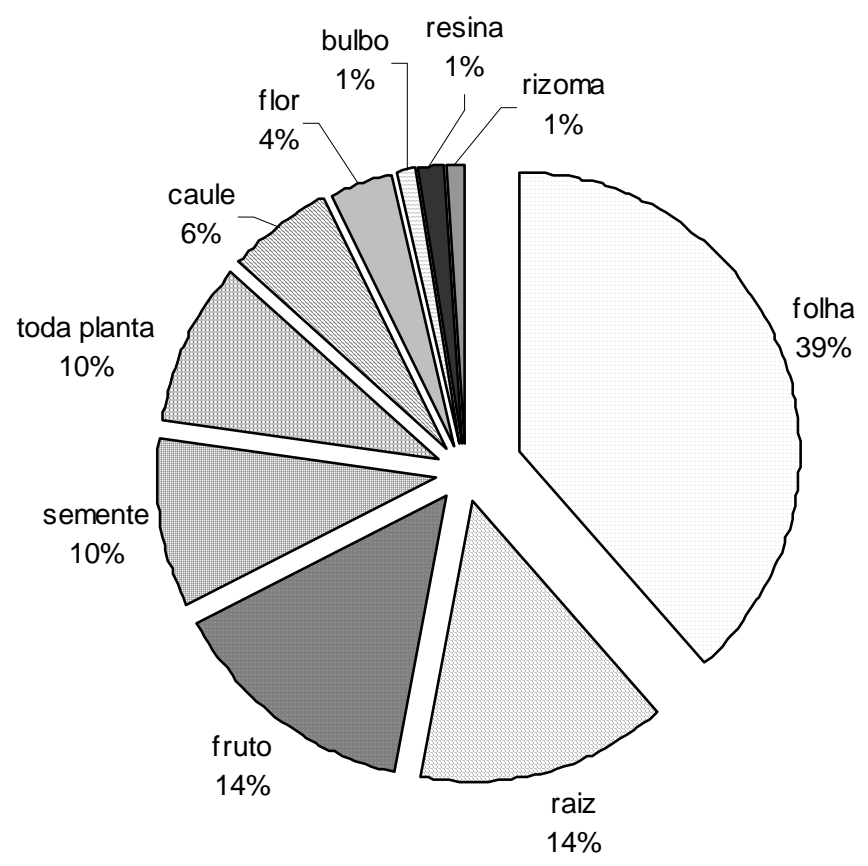

Figura 03 - Percentual em que cada órgão da planta é utilizado nas preparações medicinais.

Estudo de Cavalli et al (2007) demonstrou que o extrato bruto da raiz de Arctium minus (bardana), diminuiu os níveis plasmáticos de glicose, com potência semelhante ao medicamento sintético glibenclamida. Kiss et al (2005) atestaram que o extrato aquoso de Allium sativum (alho) mostrou-se eficiente nas condições experimentais analisadas, podendo ser utilizado como terapia complementar em pacientes diabéticos. Experimentos de Xavier (1967) comprovaram que o extrato de Baccharis trimera (carqueja) reduz o teor de glicose no sangue.

Resultados obtidos por Lino et al (2004) apontam para a validade do uso clínico de Bauhinia forficata (patade-vaca) no tratamento do diabetes tipo 2. Arduíno e Soares (1951) mostraram, em estudo clínico, o efeito hipoglicemiante da casca de Anacardium occidentale (cajueiro).

As plantas utilizadas como hipoglicemiantes na medicina popular brasileira foram tabuladas em ordem alfabética dos nomes vernaculares, acrescidos do nome científico, da família e do órgão vegetal utilizado nas preparações fitoterápicas, bem como das referências bibliográficas, onde consta o uso antidiabético do vegetal (Apêndice 01).

\section{CONCLUSÕES}

Pelo levantamento etnobotânico realizado, foram identificadas 65 espécies de vegetais utilizados pelas comunidades tradicionais pelas suas propriedades terapêuticas para o diabetes. Dessas, 5 espécies (Arctium minus, Allium sativum, Baccharis trimera, Bauhinia forficata e Anacardium occidentale) têm comprovação científica dos seus efeitos hipoglicemiantes.

O número de espécies catalogadas demonstra a importância da utilização dos fitoterápicos na medicina popular. Devem ser intensificadas as pesquisas multidisciplinares que visem comprovar a ação terapêutica de princípios ativos de origem vegetal, garantindo a segurança e eficácia na sua aplicação por parte dos profissionais de saúde.

\section{REFERÊNCIAS}

1. ALBUQUERQUE, J. M. Plantas medicinais de uso popular. Brasília: ABEAS, 1989.

2. ALMEIDA, R. N. Avaliação da atividade hipoglicemiante e isolamento de alguns triterpenóides de Bumelia sartorum Mart. 1982. 183 f. Dissertação (Mestrado) - Universidade Federal da Paraíba, João Pessoa, 1982. 
Borges, K. B. et al./Revista Eletrônica de Farmácia Vol 5(2), 12-20, 2008.

3. AGRA, M. Plantas da medicina popular dos cariris velhos. João Pessoa: União, 1996.

4. ANTOL, M. A. Te quiero: el arte de vivir mejor con las infusiones. Barcelona: Martínez Roca, 1998.

5. ARDUINO, F. H.; SOARES, M. L. Ação hipoglicemiante do cajueiro em indivíduos normais. Brasil Médico, v. 65, p. 305-308, 1951.

6. BALBACH, A. A flora nacional na medicina doméstica. 9. ed. São Paulo: Edificações do Lar, 1989.

7. BAHIA. Inventário de plantas medicinais do estado da Bahia. Salvador: SEPLANTEC, 1979.

8. BONÉ, K. Turmeric-the spice of life? British Journal of Phytotherapy, v. 2, n. 2, p. 51-60, 1991.

9. BOTSARIS, A. S. As fórmulas mágicas das plantas. 2. ed. São Paulo: Record, 1997.

10. BOWN, D. The herb society of America: encyclopedia of herb \& their uses. New York: Dorling Kindersley Publishing Inc., 1995.

11. BRAGANÇA, L. A. R. Plantas antidiabéticas: uma abordagem multidisciplinar. São Paulo: EDUFF, 1997.

12. BRUNETON, J. Pharmacognosy, Phytochemistry, medical plants. Paris: TEC \& DOC, 1995.

13. CARIBE, J.; CAMPOS, J. M. Plantas que ajudam o homem: guia prático para a época atual. São Paulo: Pensamento, 1991.

14. CAVALLI, V. L.; SORDI, C.; TONINI, K.; GRANDO, A.; MUNERON, T.; GUIGI, A.; ROMAN JUNIOR, W.A. Avaliação in vivo do efeito hipoglicemiante de extratos obtidos da raiz e folha de bardana Arctium minus (Hill.) Bernh. Brazilian Journal of Pharmacognosy, v. 17, n. 1, p. 64-70, 2007.

15. CHOPRA, D.; SIMON, D. O guia Deepak Chopra de ervas. 2. ed. Rio de Janeiro: Campus, 2001.

16. CORRÊA, A. D.; SIQUEIRA-BATISTA, R.; QUINTAS, L. E. M. Plantas medicinais: do cultivo à terapêutica. Petrópolis: Vozes, 1998.

17. CRAVO, A. B. Frutas e ervas que curam. Curitiba: Hemus, 2000.

18. CRUZ, G. L. Dicionário das plantas úteis do Brasil. Rio de Janeiro: Civilização Brasileira, 1985.

19. DIMETICH, G. S.; RANDAU, K. P.; EANDERLEY, A. G.; XAVIER, H. S. Perfil cromatográfico de duas espécies de Cissus (Vitaceae). In: SIMPÓSIO DE PLANTAS MEDICINAIS DO BRASIL, 16., 2000, Recife. Resumos... Recife: Sociedade Brasileira de Plantas Medicinais, 2000. p. 168-169.

20. FRANCO, I. J.; FONTANA, V. L. Ervas e plantas: a medicina dos simples. 7. ed. Rio Grande do Sul: Livraria Vida, 2002. 
Borges, K. B. et al./Revista Eletrônica de Farmácia Vol 5(2), 12-20, 2008.

21. FROES, V.; ROCHA, A. Alquimia vegetal. Rio de Janeiro: Record, 1997.

22. GRASSELLI, C. S. M. Uso do amido dos frutos da lobeira (Solanum lycocarpum St.-Hil.) por indivíduos diabéticos: relato de caso. Rev. Bras. Nutr. Clin, v. 16, p. 34-37, 2001.

23. GRENAND, P.; MORETTI, C.; JACCQUEMIN, H. Pharmacopées traditionelles en Guyane: Créoles, Palikur, Wayâni. Paris: ORSTROM Coll., 1987.

24. KEMBER, M.; RENG, E. Plantas medicinales de uso popular en la Amazonia Peruana. Lima: AECI/HAP, 1995.

25. KISS, A. C. I.; TAKAKU, M.; DAMASCENO, D.C.; CAMPOS K. E.; SINZATO, Y. K.; LIMA, P. O.; VOLPATO, G. $T$. Efeito do extrato aquoso de Allium sativum $L$. sobre parâmetros bioquímicos de ratas com diabete induzido por Streptozotocin. Rev. Bras. PI. Med., Botucatu, v. 8, n. 3, p. 24-30, 2006.

26. LINO, C. S.; DIOGENES, J. P.; PEREIRA, B. A.; FARIA, R. A.; ANDRADE-NETO, M.; ALVES, R. S.; QUEIROZ, M. G. Antidiabetic activity of Bauhinia forficata extracts in alloxan-diabetic rats. Biol. Pharm. Bull., v. 27, n. 1, p. 125-127, 2004.

27. LORENZI, H.; MATOS, F. J. A. Plantas medicinais no Brasil: exóticas e cultivadas. São Paulo: Instituto Plantarum, 2002.

28. MARTINS, E. R.; CASTRO, D. M.; CASTELLANI, D. C.; DIAS, J. E. Plantas medicinais. Viçosa: EUFV, 2000.

29. MATOS, F. J. A. O formulário fitoterápico do professor Dias da Rocha. Fortaleza: EUFC, 1997.

30. MATOS, F. J. A. Farmácias vivas: sistema de utilização de plantas medicinais projetado para pequenas comunidades. 3. ed. Fortaleza: EUFC, 1998.

31. MATOS, F. J. A. Plantas medicinais: guia de seleção e emprego de plantas usadas em fitoterapia no nordeste do Brasil. 2. ed. Fortaleza: EUFC, 2000.

32. MINTO, A. B. M.; CASTILHO, R. O.; GUIMARÃES, U. P.; PEREIRA, N. A.; KAPLAN, M. A. C. Atividade hipoglicemiante dos extratos de Chrysobalanus icacco L. (Chrysobalanaceae). In: SIMPÓSIO DE PLANTAS MEDICINAIS DO BRASIL, 16., 2000, Recife. Resumos... Recife: Sociedade Brasileira de Plantas Medicinais, 2000. p. 203.

33. MORS, W. B.; RIZZINI, C. T.; PERREIRA, N. A. Medicinal plants of Brazil. Michigan: Reference Publications Inc. Algonac, 2000.

34. NEGRI, G. Diabetes melito: plantas e princípios ativos naturais hipoglicemiantes. Revista Brasileira de Ciências Farmacêuticas, v. 41, n. 2, p. 121-142, 2005.

35. PANIZZA, S. Plantas que curam: cheiro de mato. 4. ed. São Paulo: IBRASA, 1997.

36. PRESTA, G. A. Interferência do chá de abagerú (Chrysobalanus icacco L.) na glicemia de jejum de camundongos e indivíduos com Diabetes mellitus-Tipo II. 1986. 173 f. Dissertação (Mestrado) - Universidade Federal do Rio de Janeiro, Rio de Janeiro, 1986. 
Borges, K. B. et al./Revista Eletrônica de Farmácia Vol 5(2), 12-20, 2008.

37. PRESTA, G. A.; PEREIRA, N. A. Atividade de abagerú (Chrysobalanus icacco L.) em modelos experimentais para o estudo de plantas hipoglicemiantes. Rev. Bras. Farm., v. 68, p. 91-101, 1987.

38. RAMOA, A. S. S.; RODRIGUES, P. C. A. Efeito da infusão de Calophyllum brasiliense na glicemia de ratos. Rev. Bras. Biol., v. 37, p. 147-149, 1997.

39. RODRIGUES, V. E. G.; CARVALHO, D. A. Plantas medicinais no domínio do cerrado. Lavras: EUFLA, 2001.

40. SELEÇÕES DIGEST READER'S. Segredos e virtudes das plantas medicinais. Rio de Janeiro, 1999.

41. SHEELA, C. G.; KUMUD, K.; AUGUSTI, K. T. Anti-diabetic effects of onion and garlic sulfoxide amino acids in rats. Planta Med., v. 61, n. 4, p. 356-357, 1995.

42. SIMÕES, C. M. O.; MENTZ, L. A.; SCHENKEL, E. P.; IRGANG, B. E.; STEHMANN, J. R. Plantas da medicina popular no Rio Grande do Sul. 5. ed. Porto Alegre: EUFRGS, 1986.

43. SOCIEDADE BRASILEIRA DE DIABETES. Tratamento e acompanhamento do diabetes mellitus: diretrizes da Sociedade Brasileira de Diabetes. Rio de Janeiro, 2008.

44. TESKE, M.; TRENTINI, A. M. M. Compêndio de Fitoterapia. 2. ed. Curitiba: Herbarium, 1995.

45. VAN DEN BERG, M. E. Plantas medicinais na Amazônia: contribuição ao seu conhecimento sistemático. Belém: Museu Paranaense Emílio Goeldi, 1993.

46. VOLPATO, G. T.; DAMASCENO, D. C.; CALDERON, I. M. P.; RUDGE, M. V. C. Revisão de plantas brasileiras com comprovado efeito hipoglicemiante no controle do Diabetes mellitus. Rev. Bras. PI. Med., Botucatu, v. 4, n. 2 , p. 35-45, 2002.

47. XAVIER, A. A. Effect of an extract of Baccharis genistelloides on the glucose level of the blood. C. R. Sciences of Society Biology and Philosophy, n.161, p. 972-974, 1967. 
Borges, K. B. et al./Revista Eletrônica de Farmácia Vol 5(2), 12-20, 2008.

\section{APÊNDICE 01: PLANTAS UTILIZADAS POPULARMENTE COMO ANTIDIABÉTICAS.}

Folha ( $F)$, casca $(C)$, raiz $(R)$, flor ( $F L)$, rizoma (RIZ), semente (S), toda planta (TP), bulbo (B), fruto (FR), resina (RE). Na coluna 5 (autores) os números correspondem à numeração das referências.

\begin{tabular}{|c|c|c|c|c|c|}
\hline $\begin{array}{l}\text { Nome(s) } \\
\text { vernacular(es) }\end{array}$ & Nome científico & Família & $\begin{array}{l}\text { Parte(s) } \\
\text { utilizada(s) }\end{array}$ & Autores & $\begin{array}{l}N^{0} \text { de } \\
\text { citações }\end{array}$ \\
\hline $\begin{array}{l}\text { Abagerú, abagem, } \\
\text { ajurú }\end{array}$ & Chrysobalanus icacco L. & Chrysobalanaceae & $\mathrm{F}$ & $11,27,32,36,37$ & 5 \\
\hline Agrião & Nasturtium officinale R. Br. & Brassicaceae & $\mathrm{F}$ & $11,13,16,27,35$ & 5 \\
\hline Alcachofra & Cynara scolymus L. & Asteraceae & $\mathrm{F}$ & $7,13,20,44$ & 4 \\
\hline Alecrim & Rosmarinus officinalis L. & Lamiaceae & $\mathrm{F}$ & 13 & 1 \\
\hline Alecrim-de-angola & Vitex agnus-castus L. & Verbenaceae & $\mathrm{F}$ & $1,10,27$ & 3 \\
\hline Alho & Allium sativum L. & Liliaceae & B & $\begin{array}{l}4,9,11,12,16,18,25 \\
27,41,44\end{array}$ & 10 \\
\hline Amalaki & Emblica officinalis Gaertn. & Euphorbiaceae & FR & 15 & 1 \\
\hline Aveia & Avena sativa $\mathrm{L}$. & Poaceae & $\mathrm{F}$ & $13,18,39,44$ & 4 \\
\hline Bálsamo & Myroxylon peruiferum L.f. & Fabaceae & RES & 6 & 1 \\
\hline Barbatimão & $\begin{array}{l}\text { Stryphnodendron } \\
\text { barbatimam Mart. }\end{array}$ & Fabaceae & C & 11,16 & 2 \\
\hline Bardana & Arctium minus (Hill) Bernh. & Asteraceae & $\mathrm{R}, \mathrm{F}$ & $11,14,27,28,35,40,44$ & 7 \\
\hline Beldroega & Portulaca oleracea L. & Portulacaceae & $F$ & 27 & 1 \\
\hline Café & Coffea arabica L. & Rubiaceae & FR, S & 11,35 & 2 \\
\hline Cajueiro & Anacardium occidentale L. & Anacardiaceae & $C, F, F R$ & $\begin{array}{l}5,7,11,13,16,20 \\
27,29,35,39,47\end{array}$ & 11 \\
\hline Camapú & Physalis angulata L. & Solanaceae & $\mathrm{R}$ & 24,27 & 2 \\
\hline Cambucá & Eugenia edulis Kiaersk. & Myrtaceae & $\mathrm{F}$ & 11 & 1 \\
\hline Cana-de-macaco & Costus spicatus Sw. & Costaceae & $\mathrm{RIZ}, \mathrm{F}$ & 27 & 1 \\
\hline Carambola & Averrhoa carambola L. & Oxalidaceae & FR & $11,27,35$ & 3 \\
\hline Carqueja & Baccharis trimera DC. & Asteraceae & $\mathrm{F}$ & $\begin{array}{l}6,7,11,13,16,17,18 \\
27,28,35,47\end{array}$ & 11 \\
\hline Cássia-fistula & Cassia fistula L. & Fabaceae & $\mathrm{R}, \mathrm{S}$ & 27 & 1 \\
\hline Cebola & Allium cepa L. & Liliaceae & $\mathrm{B}$ & 16,35 & 2 \\
\hline $\begin{array}{l}\text { Curcuma, açafrão-1 } \\
\text { terra }\end{array}$ & Curcuma longa L. & Zingiberaceae & $\mathrm{R}$ & $8,15,27,30$ & 4 \\
\hline Damiana & Turnera diffusa Willd. ex Sc] & Turneraceae & $\mathrm{F}$ & $6,7,11,16,18$ & 5 \\
\hline Dente-de-leão & $\begin{array}{l}\text { Taraxacum officinale } \\
\text { Weber }\end{array}$ & Asteraceae & $\mathrm{F}$ & $11,16,20,28,35$ & 5 \\
\hline Embaúba & Cecropia sp. & Cecropiaceae & $\mathrm{F}$ & 16,35 & 2 \\
\hline Estévia & Stevia rebaudiana Bertoni & Asteraceae & $\mathrm{F}$ & $11,16,28,35$ & 5 \\
\hline Eucalipto & Eucaliptus globulus Labill. & Myrtaceae & $\mathrm{F}$ & $6,7,9,11,16,28,42$ & 7 \\
\hline $\begin{array}{l}\text { Fafia, ginseng- } \\
\text { brasileiro }\end{array}$ & Pfaffia paniculata Kuntze & Amaranthaceae & $\mathrm{R}$ & $11,17,27,44$ & 4 \\
\hline Feijão & Phaseolus vulgaris L. & Fabaceae & $\mathrm{F}$ & $13,18,20,35$ & 4 \\
\hline Ginseng & Panax ginseng C.A.Mey. & Araliaceae & $\mathrm{R}$ & $4,9,11,44$ & 4 \\
\hline Glucomannan & $\begin{array}{l}\text { Amorphophallus konjac } \\
\text { K.Koch. }\end{array}$ & Araceae & $\mathrm{R}$ & 44 & 1 \\
\hline Guanandi & $\begin{array}{l}\text { Calophyllum brasiliense } \\
\text { Camb. }\end{array}$ & Clusiaceae & $F, C$ & $23,27,38,45$ & 4 \\
\hline Guar & $\begin{array}{l}\text { Cyamopsis tetragonolobus } \\
\text { Taub. }\end{array}$ & Fabaceae & S & 44 & 1 \\
\hline Gurmar & Gymnema sylvestre R.Br. & Fabaceae & $F$ & 15 & 1 \\
\hline Graviola & Annona muricata L. & Annonaceae & $\mathrm{F}, \mathrm{FR}, \mathrm{R}$ & $11,17,20,45$ & 4 \\
\hline
\end{tabular}


Borges, K. B. et al./Revista Eletrônica de Farmácia Vol 5(2), 12-20, 2008.

\begin{tabular}{|c|c|c|c|c|c|}
\hline Inhame & $\begin{array}{l}\text { Dioscorea dodecaneura } \\
\text { Vell. }\end{array}$ & Dioscoreaceae & $\mathrm{R}$ & 6 & 1 \\
\hline Ipê-roxo & $\begin{array}{l}\text { Tabebuia avellanedae Lorentz } \\
\text { Griseb. }\end{array}$ & Bignoniaceae & $\mathrm{F}, \mathrm{C}$ & $4,16,17$ & 3 \\
\hline Insulina-vegetal & $\begin{array}{l}\text { Cissus verticillata } \\
\text { (L.) Nicolson \& C.E.Jarvis }\end{array}$ & Vitaceae & $\mathrm{F}$ & 19,27 & 2 \\
\hline Jaborandi & $\begin{array}{l}\text { Pilocarpus jaborandi } \\
\text { Holmes }\end{array}$ & Rutaceae & $\mathrm{F}$ & $6,11,16$ & 3 \\
\hline Jambo & Eugenia jambosa Crantz & Myrtaceae & $\mathrm{FR}, \mathrm{S}$ & $7,11,16,18$ & 4 \\
\hline Jamelão, jambolão & Syzygium jambolanum DC. & Myrtaceae & FR, S & $7,11,16,18,20,30,40$ & 7 \\
\hline Jurubeba & Solanum paniculatum L. & Solanaceae & $\mathrm{R}, \mathrm{F}, \mathrm{FR}$ & $6,7,16,42,44$ & 5 \\
\hline Lobeira & $\begin{array}{l}\text { Solanum lycocarpum } \\
\text { A.St.-Hil. }\end{array}$ & Solanaceae & FR & 22,27 & 2 \\
\hline Manjericão-santo & Ocimum sanctum L. & Lamiaceae & $\mathrm{F}$ & 15 & 1 \\
\hline $\begin{array}{l}\text { Mastruço, erva-de- } \\
\text { santa-maria, }\end{array}$ & Coronopus didymus Sm. & Brassicaceae & $F, F L, S$ & 27,35 & 2 \\
\hline $\begin{array}{l}\text { Melão-de-são } \\
\text { caetano }\end{array}$ & Mormodica charantia L. & Cucurbitaceae & FR & $9,11,27,28,33,40$ & 6 \\
\hline Mucuna & Mucuna pruriens DC. & Fabaceae & $\mathrm{FR}$ & 15 & 1 \\
\hline Nogueira & Juglans regia L. & Juglandaceae & $\mathrm{F}, \mathrm{FR}$ & $11,13,40,44$ & 4 \\
\hline Oiticica & Licania rigida Benth. & Chrysobalanaceae & $\mathrm{F}$ & 3,27 & 2 \\
\hline Pata-de-vaca & Bauhinia forficata Link. & Fabaceae & $F$ & $\begin{array}{l}6,7,11,13,16,17,18, \\
20,21,26,27,30,31,35, \\
42,44\end{array}$ & 17 \\
\hline Pau-ferro, jucá & Caesalpinia ferrea Mart. & Fabaceae & TP & $6,11,16,20,29,44$ & 6 \\
\hline Pedra-hume-caá & Myrcia multiflora DC. & Myrtaceae & TP & $6,11,16,18$ & 4 \\
\hline Picão & Bidens pilosa L. & Asteraceae & $\mathrm{TP}$ & $6,7,13,16,20,28,42$ & 7 \\
\hline Pitanga & Eugenia uniflora L. & Myrtaceae & $\mathrm{F}$ & 42 & 1 \\
\hline Philium & Plantago psyllium L. & Plantaginaceae & $\mathrm{S}$ & 42,44 & 2 \\
\hline Quebra-pedra & Phyllanthus niruri $L$. & Euphorbiaceae & TP & $\begin{array}{l}6,7,11,13,15,17,18 \\
39,42,44\end{array}$ & 10 \\
\hline Quixaba & $\begin{array}{l}\text { Sideroxylon obtusifolium } \\
\text { (Roem. \& Schult.) T.D. } \\
\text { Penn. }\end{array}$ & Sapotaceae & $C, R$ & $2,3,7,11,27$ & 5 \\
\hline Romã & Punica granatum L. & Myrtaceae & TP & 11,16 & 2 \\
\hline Sabugueiro & Sambucus nigra L. & Caprifoliaceae & $\mathrm{F}, \mathrm{FI}$ & 16,20 & 2 \\
\hline Sacaca, cajuçara & Croton cajucara Benth. & Euphorbiaceae & TP & 27,45 & 2 \\
\hline Sálvia & Salvia officinalis L. & Lamiaceae & $\mathrm{F}, \mathrm{FL}$ & $11,16,18,27,28,42,44$ & 7 \\
\hline Sucupira-branca & Pterodon emarginatus Vog. & Fabaceae & $\mathrm{R}$ & $11,27,33$ & 3 \\
\hline Sucupira-do-campc & Bowdichia virgilioides H.B. & Fabaceae & C, S & 6,44 & 2 \\
\hline Urtiga & Urtica spp. & Urticaceae & TP & $\begin{array}{l}5,6,11,16,18,20,28 \\
42,44\end{array}$ & 9 \\
\hline Vassourinha & Scoparia dulcis L. & Scrophulariaceae & $\mathrm{TP}$ & $11,27,29,30$ & 4 \\
\hline
\end{tabular}

